\title{
Influence of the Maximum Blur Radius on Depth Sensor Based on Liquid Crystal Lens
}

\author{
Liming ZHENG, Xiaoxi CHEN, Yalei ZHANG, and Mao YE* \\ School of Optoelectronic Science and Engineering, University of Electronic Science and Technology of China, Chengdu \\ 611731, China \\ ${ }^{*}$ Corresponding author: Mao YE_ E-mail: mao_ye@uestc.edu.cn
}

\begin{abstract}
This paper describes how the maximum blur radius affects the depth results by depth from the defocus (DFD) method based on liquid crystal (LC) lens. Boundary frequency is determined by the maximum blur radius. It is found that if the maximum blur radius used in the calculation is larger than the real value, the depth resolution obtained is reduced; on the other hand, if one smaller than the real value is used, the depth resolution in the middle range of the scene is increased, but errors occur in the near and far planes. Using the maximum blur radius close to the real one results in the best depth results.
\end{abstract}

Keywords: Depth from defocus; liquid crystal lens; maximum blur radius

Citation: Liming ZHENG, Xiaoxi CHEN, Yalei ZHANG, and Mao YE, “'Influence of the Maximum Blur Radius on Depth Sensor Based on Liquid Crystal Lens," Photonic Sensors, 2021, 11(3): 371-375.

\section{Introduction}

Determining distance of objects from a camera is an important problem in computer vision and attracting more and more researchers' attention. Methods of depth measurement can be broadly classified into active and passive ones. Active methods include time-of-flight [1] and structured light [2], which rely on a set of optical signal transmitting and receiving devices to recover depth. Passive methods rely on algorithms that get local correspondences between two or more images. The basic idea for depth from focus (DFF) approaches is to derive depth from a series of differently focused images [3-5]. The depth at each pixel is determined by finding the focal distance at which the contrast measurement is the maximum. Depth from defocus (DFD) differs from DFF. DFD tries to derive the depth through calculating the spatially varying blur and uses only two images with different camera parameters such as focal length, aperture diameter, and image sensor position with respect to the lens [6-8].

In 1979, Sato [9] firstly proposed the concept of liquid crystal (LC) lens and fabricated optical lens using LC material. The great feature of the LC lens is that the focal length is electrically tunable and it can be driven to work as positive or negative lens [10]. Using LC lens as focusing device in the imaging system has been widely researched [11-15]. Recently, we have reported the DFD method based on an imaging system with a liquid crystal (LC) lens for focus tuning [16], which is currently being performed by mechanical movements of lens or sensor. The maximum blur radius is important in the DFD method because it determines the boundary

Received: 5 June 2020 / Revised: 5 September 2020

(C) The Author(s) 2021. This article is published with open access at Springerlink.com

DOI: $10.1007 / \mathrm{s} 13320-021-0621-\mathrm{x}$

Article type: Regular 
frequency. How the maximum blur radius affects the depth maps is discussed in this paper.

\section{DFD optic model using LC lens}

Figure 1 shows the schematic of the LC lens in this work [10]. LC (HTD028200-100, $\Delta n=0.295$ at $589 \mathrm{~nm}$ ) of $30 \mu \mathrm{m}$ thickness is sealed between glass substrates 2 and 3 with a hole-patterned $\mathrm{Cr}$ electrode and a plane indium tin oxide (ITO) electrode, respectively. The diameter of hole is $2.0 \mathrm{~mm}$. Another glass substrate 1 with plane ITO electrode is placed on the top. Both substrates with ITO electrode are of $1.1 \mathrm{~mm}$ thickness. Substrate with hole-patterned electrode is of $0.7 \mathrm{~mm}$. Applying alternating current (AC) voltages $V_{1}$ and $V_{2}$ of $1 \mathrm{kHz}$ across electrodes will produce spatially uniform and axially symmetrical electric field in the LC layer and form lens-like refractive index distribution. LC lens optical property at different voltages can be measured through interferometer [17]. Partial results are summarized in Table 1. The rms aberration remains below 0.1 wave and $\mathrm{LC}$ lens can work in both negative and positive lens states.

Table 1 Optical property of LC lens at different voltages.

\begin{tabular}{cccc||cccc}
\hline$V_{1}\left(V_{\mathrm{rms}}\right)$ & $V_{2}\left(V_{\mathrm{rms}}\right)$ & $P\left(\mathrm{~m}^{-1}\right)$ & $\mathrm{rms}$ & $V_{1}\left(V_{\mathrm{rms}}\right)$ & $V_{2}\left(V_{\mathrm{rms}}\right)$ & $P\left(\mathrm{~m}^{-1}\right)$ & $\mathrm{rms}$ \\
\hline 28 & 12 & 2.7 & 0.08 & 10 & 49 & -4.2 & 0.05 \\
28 & 14 & 2.8 & 0.06 & 12 & 49 & -4.0 & 0.05 \\
28 & 16 & 2.8 & 0.04 & 14 & 49 & -3.4 & 0.05 \\
28 & 18 & 2.8 & 0.04 & 16 & 49 & -3.0 & 0.04 \\
28 & 20 & 2.5 & 0.03 & 18 & 49 & -2.6 & 0.04 \\
28 & 22 & 2.2 & 0.04 & 20 & 49 & -2.3 & 0.04 \\
28 & 24 & 2.0 & 0.04 & 22 & 49 & -2.0 & 0.03 \\
28 & 26 & 1.7 & 0.03 & 24 & 49 & -1.6 & 0.03 \\
28 & 28 & 1.5 & 0.03 & 26 & 49 & -1.2 & 0.03 \\
28 & 30 & 1.2 & 0.03 & 28 & 49 & -0.9 & 0.03 \\
28 & 32 & 0.9 & 0.03 & 30 & 49 & -0.6 & 0.03 \\
28 & 34 & 0.7 & 0.02 & 32 & 49 & -0.4 & 0.03 \\
\hline
\end{tabular}

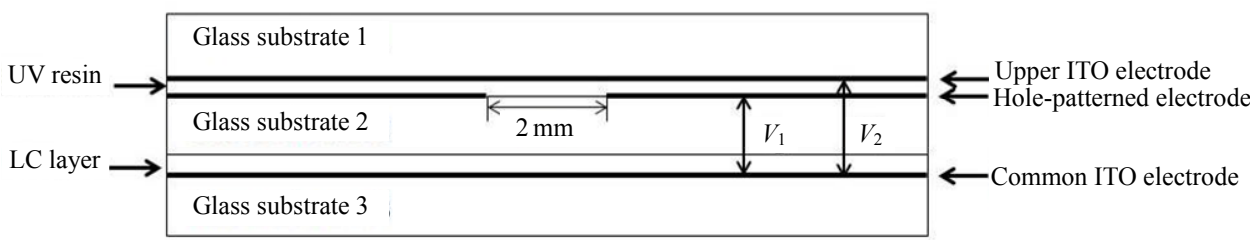

Fig. 1 Schematic diagram of LC lens.

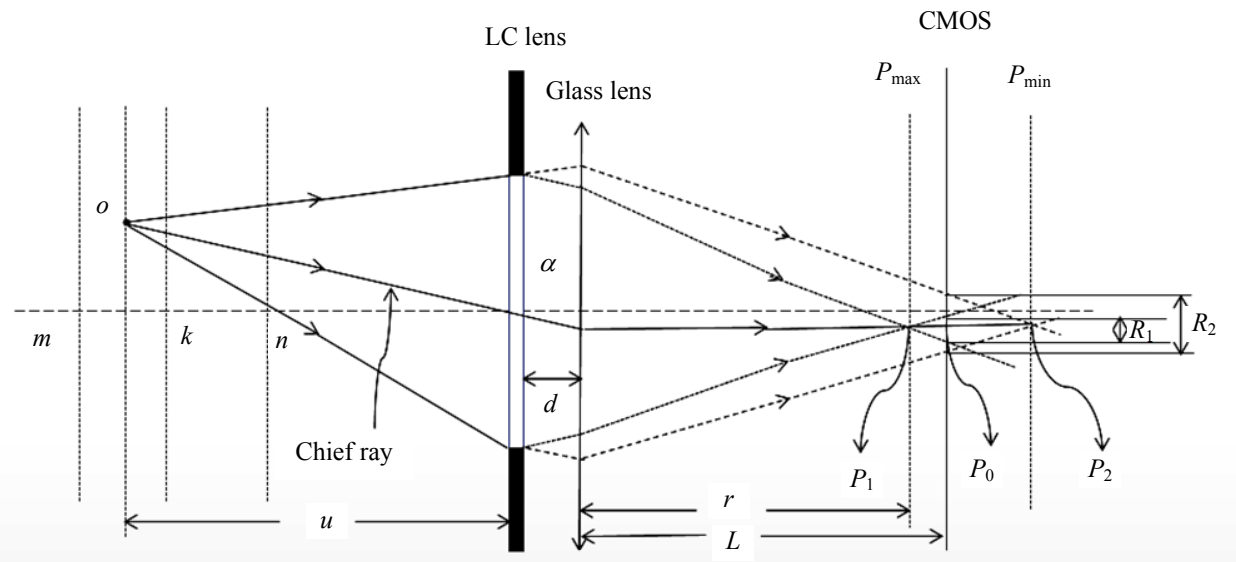

Fig. 2 Image formation with LC lens.

The imaging system is shown in Fig. 2. It consists of an LC lens and a glass lens. Let the optical power of glass lens, LC lens, and the distance between the LC lens and the glass lens be $P_{g}, P_{\mathrm{LC}}$, and $d$, respectively. The optical power $P$ of the imaging system varies from $P_{\min }$ to $P_{\max }$ as $P_{\mathrm{LC}}$ changes from $P_{\mathrm{LC}}^{-}$to $P_{\mathrm{LC}}^{+}$, which is calculated by (1) and (2). The initial focal plane is $k$ where the LC lens is in the non-lens state. The depth measurement range is $[m, n]$. If $P_{\mathrm{LC}}=P_{\mathrm{LC}}^{+}$, the system focuses on near plane $n$, and if $P_{\mathrm{LC}}=P_{\mathrm{LC}}^{-}$, on far plane $m$. 


$$
\begin{gathered}
P_{\text {min }}=P_{g}+P_{\mathrm{LC}}^{-}-d P_{g} P_{\mathrm{LC}}^{-} \\
P_{\text {max }}=P_{g}+P_{\mathrm{LC}}^{+}-d P_{g} P_{\mathrm{LC}}^{+} .
\end{gathered}
$$

In the imaging system shown in Fig. 2, LC lens plays as the diaphragm due to its aperture size is much smaller than that of glass lens. The chief ray from object point $o$ passes through the center of diaphragm and hits the complementary metal-oxide semiconductor (CMOS) plane at point $P_{0}$. When the optical power of LC lens changes to $P_{\mathrm{LC}}^{+}$or $P_{\mathrm{LC}}^{-}$, object point $o$ focuses at $P_{1}$ and $P_{2}$ and leaves blurred circular image of radius $R_{1}$ and $R_{2}$ on CMOS, respectively. The chief ray propagation direction will not change although the optical power of LC lens changed, so the center of the blurred circular image will always be $P_{0}$. The image magnification remains constant.

We acquire depth through the DFD algorithm based on rational filters $[6,16]$. The power required to focus on any plane $o$ in the range $[m, n]$ is

$$
P=\frac{\mu\left(P_{\max }-P_{\min }\right)}{2}+\frac{P_{\max }+P_{\min }}{2}
$$

where parameter $\mu \in[-1,1]$ is the normalized depth value. For example, if system focuses on plane $n$, the power required is $P_{\max }$, and correspondingly $\mu=1$. When $P_{\mathrm{LC}}=P_{\mathrm{LC}}^{+}$, object $o$ will cause defocused spot on the sensor plane, and the radius of the defocused spot $R_{1}$ is derived from (4)-(6):

$$
\begin{aligned}
& \frac{r}{L-r}=\frac{\alpha}{R_{1}} \\
& \frac{1}{u}+\frac{1}{L}=P \\
& \frac{1}{u}+\frac{1}{r}=P_{\max } \\
& R_{1}=a L \varepsilon(1-\mu)
\end{aligned}
$$

where $a$ is the radius of the aperture of the system, $L$ is the distance between sensor plane and lens, $u$ is the object distance of object $o, r$ is the image distance of object $o$ when $P=P_{\max }$, and $\varepsilon=\left(P_{\mathrm{LC}}^{+}-P_{\mathrm{LC}}^{-}\right) / 2$. When $P_{\mathrm{LC}}=P_{\mathrm{LC}}^{-}$, the defocus radius is

$$
R_{2}=a L \varepsilon(1+\mu)
$$

We can find the maximum blur radius is $2 a L \varepsilon$. The pillbox function is used as blur function:

$$
h(x, y)=\frac{1}{\pi R^{2}} \Pi\left(\frac{1}{2 R} \sqrt{x^{2}+y^{2}}\right)
$$

where $\Pi(t)$ is rectangular function. If $t<0.5, \Pi(t)=1$, else $\Pi(t)=0 . x$ and $y$ represent pixel positions. $R$ is the defocus radius.

The Fourier transform of blur function is

$$
H(u, v)=\frac{j_{1}\left(2 \pi R \sqrt{u^{2}+v^{2}}\right)}{\pi R \sqrt{u^{2}+v^{2}}}
$$

where $u$ and $v$ represent spatial frequencies, and $j_{1}$ is the first-order Bessel function.

According to [6], the normalized ratio function is

$$
\frac{M(u, v)}{P(u, v)}=\frac{H_{1}\left(u, v ; R_{1}\right)-H_{2}\left(u, v ; R_{2}\right)}{H_{1}\left(u, v ; R_{1}\right)+H_{2}\left(u, v ; R_{2}\right)}
$$

where $M(u, v)$ denotes the subtraction of the blur function $H(u, v)$ of blur radius $R_{1}$ and $R_{2}$. $P(u, v)$ is the sum of the blur function of blur radius $R_{1}$ and $R_{2}$. We can find the normalized ratio function is a monotonic function of depth value $\mu$ if space frequency $f_{r}=\sqrt{u^{2}+v^{2}} \leq 0.61 / 2 a L \varepsilon$. The boundary frequency is $f_{\text {rmax }}=0.61 / 2 a L \varepsilon$. It means that we can make use of monotonicity to get depth value $\mu$ uniquely when space frequency $f_{r} \leq f_{\text {rmax }}$. We can find the boundary frequency $f_{\text {rmax }}$ is determined by the maximum blur radius $2 a L \varepsilon$. In this work, the computation method of DFD is based on convolution kernels, which has low computational cost and high spatial resolution. For kernels of size $M$, the discrete frequency period is $1 / M$. In order to calculate the depth value where space frequency is below $f_{\max }$, and suppress other frequency component, we set the condition as $2 / M \leq f_{\text {rmax }}$.

In theory, if $P_{\mathrm{LC}}=0, P=P_{g}$, system focuses on $k$, and if $d$ is too small to ignore, the corresponding value of $\mu$ is

$$
\mu=\left(P_{\mathrm{LC}}^{-}+P_{\mathrm{LC}}^{+}\right) /\left(P_{\mathrm{LC}}^{-}-P_{\mathrm{LC}}^{+}\right) .
$$

For visualization, we need to map depth value 
$\mu \in[-1,1]$ to $[0,255]$ using $-128 \mu+128$. The grayscale encoded depth value of the initial focus plane is

$$
-128\left(P_{\mathrm{LC}}^{-}+P_{\mathrm{LC}}^{+}\right) /\left(P_{\mathrm{LC}}^{-}-P_{\mathrm{LC}}^{+}\right)+128 .
$$

Supposing $P_{\mathrm{LC}}^{+}=-P_{\mathrm{LC}}^{-}$, the depth grayscale value of the initial focus plane should be 128 .

\section{Experimental results}

In the experiment, we select positive optical power $P_{\mathrm{LC}}^{+}=2.2\left(V_{1}=28 V_{\text {rms }}, V_{2}=22 V_{\text {rms }}\right) \quad$ and $P_{\mathrm{LC}}^{-}=-2.3\left(V_{1}=20 V_{\mathrm{rms}}, V_{2}=49 V_{\mathrm{rms}}\right)$ negative optical power to get the far and near focused images. The LC lens sticks to the convex lens of $8.0 \mathrm{~mm}$ focal length and its aperture is used as the aperture stop of the compound lens. The distance $d$ between the LC lens and the glass lens is negligible. The magnification of the same object point remains unchanged under different LC lens powers. The images resolution is $1280 \times 960$ and pixel size is $2.2 \mu \mathrm{m}$. There are 7 objects in the scene placed with different distances. First, tune the system to focus at $u_{0}=300 \mathrm{~mm}$ when the LC lens is in the non-lens state. Then, tune the LC lens working on the optical power $P_{\mathrm{LC}}^{+}$and $P_{\mathrm{LC}}^{-}$, respectively, to acquire two images which focus on the nearest and farthest object, as shown in Figs. 3(a) and 3(b). The radius of the aperture is $2.0 \mathrm{~mm}$. We can easily get the maximum blur radius of the imaging system form $2 a L \varepsilon$ is $R=16.8$ pixels. The depth map, as shown in Fig. 4, is derived from the two images. Objects with different distances show different grayscale values, and the smaller the value is, the closer it is to the imaging system. The depth grayscale values and the distance of each object are summarized in Table2. The grayscale depth value of the initial focus plane is 121 , which is near 128 .

Table 2 Object distance and depth value.

\begin{tabular}{ccc}
\hline Object & $\mu(\mathrm{mm})$ & Depth value \\
\hline 1 & 180 & 7 \\
2 & 240 & 89 \\
3 & 300 & 121 \\
4 & 370 & 169 \\
5 & 490 & 185 \\
6 & 630 & 206 \\
7 & 820 & 218 \\
\hline
\end{tabular}

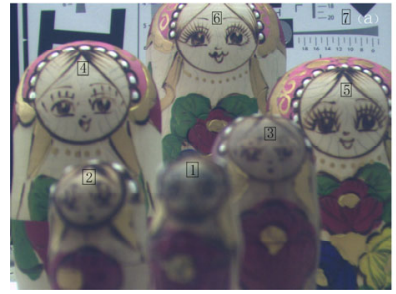

(a)

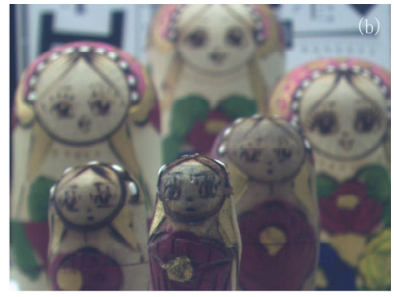

(b)
Fig. 3 Far focused and near focused images: (a) negative LC lens optical power and (b) positive LC lens optical power.

If a larger maximum blur radius $R=22.0$ pixels or a smaller maximum blur radius $R=11.7$ pixels is used, the depth maps are obtained as shown in Figs.5(a) and 5(b), respectively. From the depth map, we can still judge the distance order of objects in the scene based on the grayscale value. The grayscale depth value of different maximum blur radius has an approximately linear relationship with the reciprocal of object distance, as shown in the Fig. 6. Using the maximum blur radius larger than the actual value means that the measurement range is wider than $[m$, $n]$, which results in a narrower grayscale value of the depth to be measured, and the resolution of the depth grayscale is reduced. On the contrary, the maximum blur radius smaller than the actual value makes the depth resolution increase, but for the measurement range is narrower than $[m, n]$, more calculation errors occur near planes $m$ and $n$.

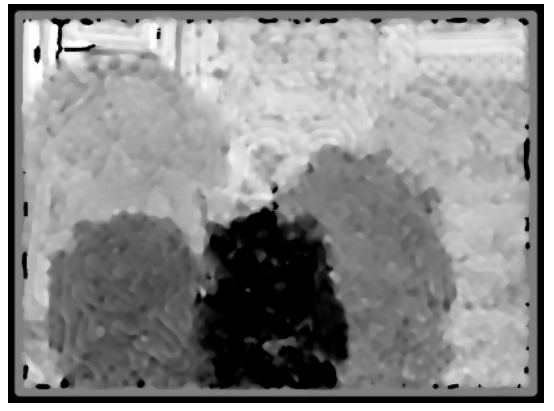

Fig. 4 Depth map with maximum blur radius of 16.8 pixels.

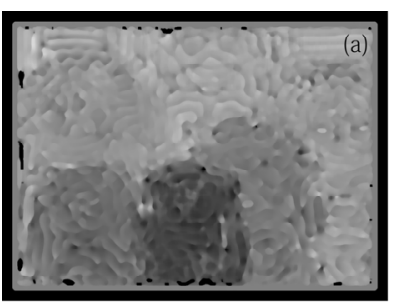

(a)

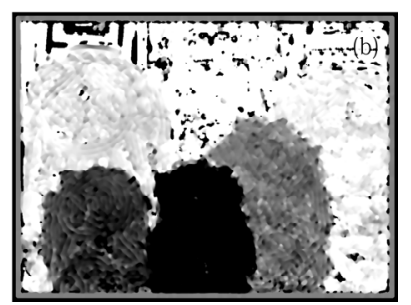

(b)
Fig. 5 Depth map: (a) maximum blur radius of 22.0 pixels, and (b) maximum blur radius of 11.7 pixels. 


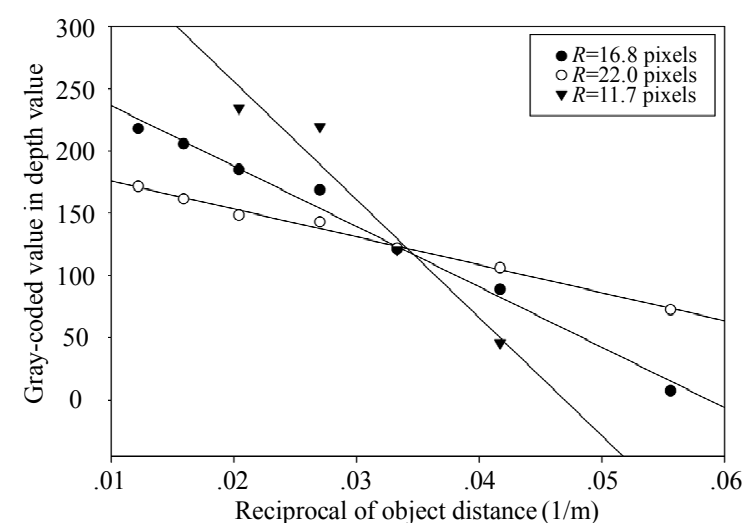

Fig. 6 Relationship between the depth value and the reciprocal of object distance.

\section{Conclusions}

This paper demonstrates how the different maximum blur radii adopted in calculation affects the depth results in the DFD method based on LC lens. The depth resolution is reduced if using a larger maximum blur radius than the actual value. Using the maximum blur radius smaller than the actual value, the depth resolution of the middle range is increased, but errors occur in the near and far planes. Using the maximum blur radius close to the actual value gets the best depth results.

\section{Acknowledgment}

This work was partially supported by Sichuan Science and Technology Program (Grant No. 20YYJC4365).

Open Access This article is distributed under the terms of the Creative Commons Attribution 4.0 International License (http://creativecommons.org/licenses/by/4.0/), which permits unrestricted use, distribution, and reproduction in any medium, provided you give appropriate credit to the original author(s) and the source, provide a link to the Creative Commons license, and indicate if changes were made.

\section{References}

[1] S. Foix, G. Alenya, and C. Torras, "Lock-in time-of-flight (ToF) cameras: a survey," IEEE Sensors Journal, 2011, 11(9): 1917-1926.

[2] E. Horn and N. Kiryati, "Toward optimal structured light patterns," Image and Vision Computing, 1999,
17(2): 87-97.

[3] J. Ens and P. Lawrence, "A matrix based method for determining depth from focus," IEEE Proceedings, 1991, 1: 600-606.

[4] J. Ens and P. Lawrence, "An investigation of method for determining depth from focus," IEEE Transactions on Pattern Analysis and Machine Intelligence, 1993, 15(2): 97-108.

[5] M. Moeller, M. Benning, C. Schoenlieb, and D. Cremers, "Variational depth from focus reconstruction," IEEE Transactions on Image Processing, 2015, 24(12): 5369-5378.

[6] M. Watanabe and S. K. Nayar, "Rational filters for passive depth from defocus," International Journal of Computer Vision, 1998, 27(3): 203-225.

[7] M. Subbarao and G. Surya, "Depth from defocus: a spatial domain approach," International Journal of Computer Vision, 1994, 13(3): 271-294.

[8] I. Blayvas, R. Kimmel, and E. Rivlin, "Role of optics in the accuracy of depth-from-defocus systems," Journal of the Optical Society of America A, 2007, 24(4): 967-972.

[9] S. Sato, "Liquid-crystal lens-cells with variable focal length," Japanese Journal of Applied Physics, 1979, 18(9): 1679

[10] B. Wang, M. Ye, and S. Sato, "Liquid crystal lens with focal length variable from negative to positive values," IEEE Photonics Technology Letters, 2005, 18(1): 79-81.

[11] M. Ye, B. Wang, and S. Sato, "Liquid-crystal lens with a focal length that is variable in a wide range," Applied Optics, 2004, 43(35): 6407-6412.

[12] M. Ye, B. Wang, M. Uchida, S. Yanase, S. Takahashi, and S. Sato, "Focus tuning by liquid crystal lens in imaging system," Applied Optics, 2012, 51(31): 7631-7634.

[13] Y. H. Lin, M. S. Chen, and H. C. Lin, "An electrically tunable optical zoom system using two composite liquid crystal lenses with a large zoom ratio," Optical Express, 2011, 19(5): 4714-4721.

[14] M. Ye, B. Wang, M. Uchida, S. Yanase, S. Takahashi, M. Yamaguchi, et al., "Low-voltage-driving liquid crystal lens," The Japan Society of Applied Physics, 2010, 49(100204): 1-4

[15] H. C. Lin and Y. H. Lin, “A fast response and large electrically tunable-focusing imaging system based on switching of two modes of a liquid crystal lens," Applied Physics Letters, 2010, 97(6): 063505

[16] M. Ye, X. X. Chen, Q. C. Li, J. Zeng, and S. D. Yu, "Depth from defocus measurement method based on liquid crystal lens," Optics Express, 2018, 26(22): 28413-28420.

[17] X. Chen, Y. Bai, C. Chao, and M. Ye, "Driving liquid crystal lens to extend focus range," Japanese Journal of Applied Physics, 2018, 57(7): 072601. 